\title{
Analysis of Earth Gravity Tide Signal Based on EMD and Information Extraction
}

\author{
Dongjun Zhong ${ }^{\mathrm{a}}$, Haiyan Quan ${ }^{\mathrm{b}}$ \\ Faculty of Information Engineering and Automation, Kunming University of Science and Technology, \\ Kunming650500, china \\ a963240382@qq.com, bquanhaiyan@163.com
}

Keywords: Empirical Mode Decomposition, the earth gravity tide signal, information.

\begin{abstract}
Empirical Mode Decomposition (EMD) is a decomposition algorithm which is used to analyze nonlinear and time-varying. Different from the traditional signal analysis method, the decomposition is data-driven and self-adaptive. EMD has been widely used in blind source separation and received attention because of its potential application in signal processing. EMD algorithm and its application in earth gravity tide signal are introduced. EMD is a potential method in the earth gravity tide signal. So we can extract useful information from it to understand earthquake precursors.
\end{abstract}

\section{Introduction}

Empirical Mode Decomposition (EMD) method is considered to be a major breakthrough of the linear and steady spectrum analysis on the basis of the Fourier transform since 2000 [1]. The method is based on time scale characteristics of the data for signal Decomposition. It dose not need to set any basis function in advance. It has essential different from the Fourier decomposition and wavelet decomposition method. Precisely because of this characteristic, the EMD method in theory can be applied to any type of signal decomposition and has very obvious advantages on the process of non-stationary and nonlinear data [2,3]. It is suitable for analyzing nonlinear and non-stationary signal sequence. The EMD method is put forward in different engineering fields quickly and efficiently used. For example in the ocean, atmosphere, astronomical observation data, seismic record analysis, mechanical fault diagnosis and so on.

The earth gravity tide is a recurring phenomenon that is caused by the tide generating force of the moon and sun $[4,5]$. The earth is not completely rigid, but solid matter between elastic and plastic body. And the gravitational pull of the moon and sun not only cause ocean tide and atmospheric tide but the earth gravity tide as well [6,7].

\section{Empirical Mode Decomposition}

Empirical Mode Decomposition algorithm is the core of the Hilbert-Huang transform (HHT) algorithm. It can make the complex signal to be decomposed into finite Intrinsic Mode Function (IMF) [8]. The each IMF component contains the local characteristics of original signals in different time scales. Empirical mode decomposition method can make non-stationary data smoothly. Then the Hilbert transform can obtain the spectrum diagram, get the frequency of the physical meaning. EMD algorithm is defined through the algorithm process, and is not defined by certain theory formula. So it is very difficult to carry on the accurate theoretical analysis. We now can only use a lot of digital simulation test to do some in-depth study of its performance. The EMD algorithm aimed that the bad performance of signal is decomposed into a set of good intrinsic Mode Function. And the IMF must meet the following two properties [9].

Signal of extreme amount (the maximum or minimum) equal to the number of passing zero or at most a difference.

Average is zero of the local maximum value and local minimum on the envelope.

The algorithm principle is the (a), (b), and (c). 
(a) Look for signals of all the maximum and minimum $x(t)$ and then use the cubic spline interpolation algorithm to get envelope $x_{h}(t)$ and the envelope $x_{l}(t)$. The signal between all data points should be located in the upper and lower envelope. At the same time, two envelope average $m(t)$ can be calculated as the (1).

$$
m(t)=\left(x_{h}(t)+x_{l}(t)\right) / 2
$$

(b) Set a variable $x_{1}(t)$, make it as follows.

$$
x_{1}(t)=x(t)-m(t)
$$

If meet the two IMF standards, it can be used as the first IMF $c_{1}(t)$, otherwise repeat (a) and (b), until they get the first component of the IMF.

(c) Calculate the residual signal.

$r_{1}(t)=x(t)-c_{1}(t)$

As a new signal, repeat (a) and (b), until all the IMF component extraction. Original data finally is decomposed into n IMF and a surplus $r_{n}(t)$.

$$
x(t)=\sum_{i=1}^{n} c_{i}(t)+r_{n}(t)
$$

Hilbert transform of each intrinsic mode components $c_{1}(t)$ is the (5).

$$
c_{i}^{\prime}(t)=\int_{\pi}^{1} \int_{-\infty}^{\infty} c_{i}(\tau) d \tau
$$

Complex signal.

$$
Z_{i}(t)=c_{i}(t)+j c_{i}^{\prime}(t)
$$

As a result, the Hilbert transform of $x(t)$ is the (7).

$$
Z(t)=x(t)+j x_{i}^{\prime}(t)==\sum_{i=1}^{n} Z_{i}(t)
$$

The modulus of $Z(t)$ is the signal envelope of $x(t)$.

\section{The Analysis of Earth Gravity Tide Signal Based on EMD}

In the study of the earth gravity tide, there are two aspects of the data. On the one hand is actual observed data, on the other theoretical calculation data. The earth gravity tidal wave is a periodic signal [10]. And its periodicity is very complex, including half-day period component, day period component and so on. Experiment data were chosen from the observation points [11].

Four months data signal is generated in Fig.1 and Fig.2. 


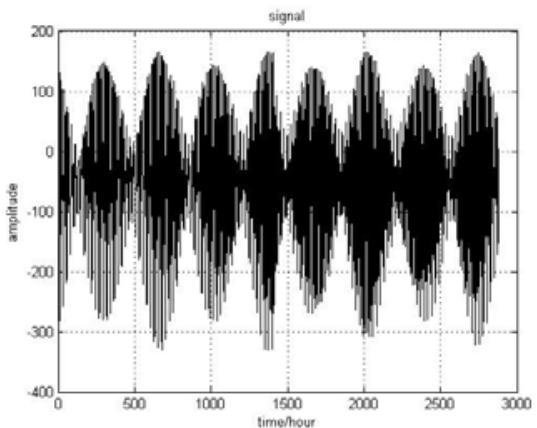

Fig.1.Input signal.

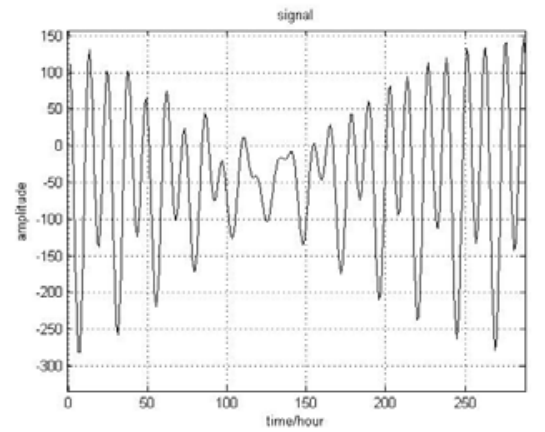

Fig.2.Local Input signal.

Figure 1 represents the earth gravity tide signal. We capture part of the signal as shown in figure 2. The input signal do EMD. We can get the EMD signals in Fig.3.

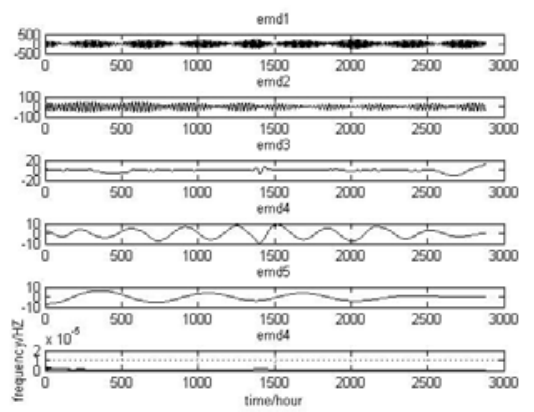

Fig.3.EMD signals.

After the emd, we got all the decomposition signals. As you can see in figure 3, the first way and the second way are obvious. So we choose the first way and the second way to do the Hilbert transform.

In Fig.4, the majority of ordinate values are small-scale fluctuations in the vicinity of 2.3148e-005 Hz. And it is the frequency of half-day period wave. So Fig.4 reflects the half-day period components.

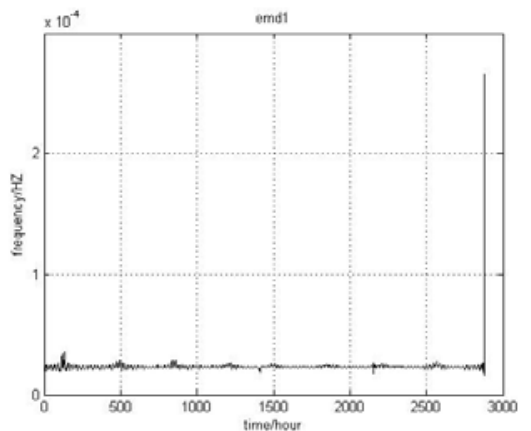

Fig.4.half-day period.

In Fig.5, the majority of ordinate values are small-scale fluctuations in the vicinity of $1.1730 \mathrm{e}-005$ $\mathrm{Hz}$. And the frequency of day period component is just in the vicinity of $1.1574 \mathrm{e}-005 \mathrm{~Hz}$. So Fig.5 reflects day period component.

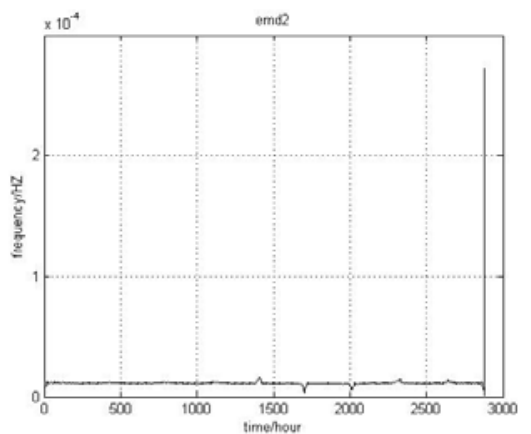

Fig.5.The day period. 
From Fig.4 and Fig.5, we can get the datum table.

Table 1 the contrast of between theoretical frequency and actual frequency.

\begin{tabular}{lll}
\hline components & half-day period & day period \\
\hline Theoretical frequency/Hz & $2.3148 \mathrm{e}-005$ & $1.1574 \mathrm{e}-005$ \\
Actual frequency/Hz & $2.3250 \mathrm{e}-005$ & $1.1730 \mathrm{e}-005$ \\
\hline
\end{tabular}

\section{Summary}

Empirical Mode Decomposition can effectively decompose the earth gravity tide signal. So the EMD analysis can apply to the earth gravity tide signal decomposition, which allows us to understand the basic information of the tidal signal and further knows that the tidal signal reflected geophysical information. Through computer simulation, it can get half-day period wave and day wave.

\section{Acknowledgment}

This work is supported by National Natural Science Foundation of China (No. 41364002), State Science Foundation of Yunnan (No. 2009ZC048M) and Science Grant of Kunming University of Science and Technology (No. KKZ3201103022). The authors are graceful for the support of the grants.

\section{References}

[1]. L. Lin, Y. Wang, and H. Zhou, "Iterative filtering as an alternative algorithm for empirical mode decomposition, " Advances in Adaptive Data Analysis, vol. 1, no. 04, pp. 543-560, 2009.

[2]. Minet C, Eineder M, Yague-Martinez N. Haiti earthquake (12.01. 2010) surface shift estimation using TerraSAR-X data[C]//Geoscience and Remote Sensing Symposium (IGARSS), 2011 IEEE International. IEEE, 2011: 2488-2491.

[3]. Baek S H, Shum C K. Antarctic ocean tide signal restoration using differential InSAR technique[C]//Synthetic Aperture Radar (APSAR), 2011 3rd International Asia-Pacific Conference on. IEEE, 2011: 1-4.

[4]. Nie B, Du J, Liu H, et al. Crowds' classification using hierarchical cluster, rough sets, principal component analysis and its combination[C]//Computer Science-Technology and Applications, 2009. IFCSTA'09. International Forum on. IEEE, 2009, 1: 287-290.

[5]. Puyati W, Walairacht A. Efficiency improvement for unconstrained face recognition by weightening probability values of modular PCA and wavelet PCA[C]//Advanced Communication Technology, 2008. ICACT 2008. 10th International Conference on. IEEE, 2008, 2: 1449-1453.

[6]. B. L. Barnhart, The Hilbert-Huang Transform: Theory, applications, development. University of Iowa, 2011.

[7]. N. E. Huang, Z. Shen, S. R. Long, M. C. Wu, H. H. Shih, Q. Zheng, N.- C. Yen, C. C. Tung, and H. H. Liu, "The empirical mode decomposition and the Hilbert spectrum for nonlinear and non-stationary time series analysis, " Proceedings of the Royal Society of London. Series A: Mathematical, Physical and Engineering Sciences, vol. 454, no. 1971, pp. 903-995, 1998.

[8]. Z. Qingjie, Z. Huayong, and S. Lincheng, "A new method for mitigation of end effect in empirical mode decomposition, " in Informatics in Control, Automation and Robotics (CAR), 2010 2nd International Asia Conference on, vol. 1. IEEE, 2010, pp. 400-403. 
[9]. L. Yang, C. Huang, and Z. Yang, "The model and construction of weak IMFs, " Computational and Applied Mathematics, pp. 1-10, 2014.

[10]. V. Vatchev and R. Sharpley, "Decomposition of functions into pairs of intrinsic mode functions, " Proceedings of the Royal Society A: Mathematical, Physical and Engineering Science, vol. 464, no. 2097, pp. 2265-2280, 2008.

[11]. Eineder M, Balss U, Gisinger C, et al. TerraSAR-X pixel localization accuracy: Approaching the centimeter level[C]//Geoscience and Remote Sensing Symposium (IGARSS), 2014 IEEE International. IEEE, 2014: 2669-2670. 